\title{
Right trisectionectomy with en bloc portal vein resection for cholangiocarcinoma after preoperative stenting for main portal vein occlusion
}

\author{
Shin Hwang ${ }^{1}$ and Gi-Young Ko ${ }^{2}$ \\ Departments of ${ }^{1}$ Surgery and ${ }^{2}$ Radiology, Asan Medical Center, \\ University of Ulsan College of Medicine, Seoul, Korea
}

\begin{abstract}
Deprivation of portal blood flow decreases the hepatic function, thus hepatobiliary cancer patients with total occlusion of the main portal vein (PV) are usually not indicated for major hepatectomy. We herein present a 37-year-old male patient with advanced intrahepatic cholangiocarcinoma, in whom right trisectionectomy was indicated. However, the main PV was nearly completely occluded by tumor invasion, thus resolution of jaundice was markedly slow. To restore the liver function through PV recanalization, a wall stent was inserted percutaneously. Jaundice resolved progressively after PV stenting. Right trisectionectomy, caudate lobectomy, bile duct resection, and en bloc PV segmental resection with iliac vein homograft interposition were performed. However, PV thrombosis developed at the site of PV stent removal, thus a new wall stent was inserted during the operation. The pathology report presented that the tumor was a $5.2 \mathrm{~cm}$-sized well-differentiated adenocarcinoma of periductal infiltrating type with lymph node metastasis. During the follow-up, the interposed PV segment with a wall stent was gradually occluded with development of portal collaterals. At 5 years after surgery, the PV stent was completely occluded and collaterals developed. The patient experienced repetition of febrile episodes of unknown causes. He is currently alive for 8 years with no evidence of tumor recurrence. The detailed surgical procedures were presented with a supplementary video clip of 5 minutes. (Ann Hepatobiliary Pancreat Surg 2020;24:174-181)
\end{abstract}

Key Words: Right trisectionectomy; Intahepatic cholangiocarcinoma; Stent; Portal vein; Interposition

\section{INTRODUCTION}

Deprivation of portal flow decreases the liver function and interferes functional recovery from obstructive jaundice. Patients with perihilar malignancy combined with total occlusion of the main portal vein (PV) are usually not indicated for major hepatectomy because major liver resection of the icteric liver incurs high risk of post-hepatectomy liver failure. If the portal blood flow is restored through percutaneous PV stenting, it can provide an opportunity to recover the liver function preoperatively and decrease the risk of post-hepatectomy liver failure. In the literature, there were only two reports on preoperative PV embolization after PV stenting in patients with gallbladder cancer and perihilar cholangiocarcinoma with severe PV tumor invasion and stenosis. ${ }^{1,2}$
We herein present a case report of successful right trisectionectomy with en bloc portal vein resection for central-type intrahepatic cholangiocarcinoma after improvement of the liver function through preoperative stenting for main portal vein occlusion. Since there was no report on intraoperative en bloc removal of the PV stent yet in the literature, this case would be the first case. The detailed surgical procedures were presented with a Supplementary video clip of 5 minutes.

\section{CASE}

The case was a 39-year-old male patient with central-type intrahepatic cholangiocarcinoma. The preplanned extent of surgery was right trisectionectomy and caudate lobe resection combined with bile duct resection. However,

Received: March 23, 2020; Revised: March 24, 2020; Accepted: March 25, 2020

Corresponding author: Shin Hwang

Department of Surgery, Asan Medical Center, University of Ulsan College of Medicine, 88 Olympic-ro 43-gil, Songpa-gu, Seoul 05505, Korea Tel: +82-2-3010-3930, Fax: +82-2-3010-6701, E-mail: shwang@amc.seoul.kr

Copyright (C) 2020 by The Korean Association of Hepato-Biliary-Pancreatic Surgery

This is an Open Access article distributed under the terms of the Creative Commons Attribution Non-Commercial License (http://creativecommons.org/ licenses/by-nc/4.0) which permits unrestricted non-commercial use, distribution, and reproduction in any medium, provided the original work is properly cited. Annals of Hepato-Biliary-Pancreatic Surgery • pISSN: 2508-5778 - eISSN: 2508-5859 
the main PV was nearly completely occluded by perihilar tumor invasion (Fig. 1A, B), thus obstructive jaundice was resolved very slowly despite endoscopic nasobiliary drainage. It took 25 days to decrease the serum total bilirubin concentration from $16 \mathrm{mg} / \mathrm{dl}$ to $7 \mathrm{mg} / \mathrm{dl}$ with endoscopic nasobiliary drainage. During this period of biliary decompression, the main PV was progressively occluded and finally nearly totally obstructed (Fig. 1C, 2A).

To restore the liver function and to facilitate biliary decompression through PV recanalization, a self-expandable wall stent of $6 \mathrm{~cm} \times 12 \mathrm{~mm}$ was inserted into the main PV up to the umbilical portion of the left PV through the left transhepatic approach (Fig. 1D, 2B). After 5 days, the serum total bilirubin level gradually decreased to $3 \mathrm{mg} / \mathrm{dl}$, thus we decided to perform radical surgery with an intention of curative treatment.

The operation began with mini-laparotomy to examine whether peritoneal seeding was present or not (Fig. 3A). The distal bile duct was dissected first and transected to assess the status of tumor invasion, in which the distal bile duct resection margin was tumor-free (Fig. 3B-D). Dissection continued toward the hepatic hilum, but the
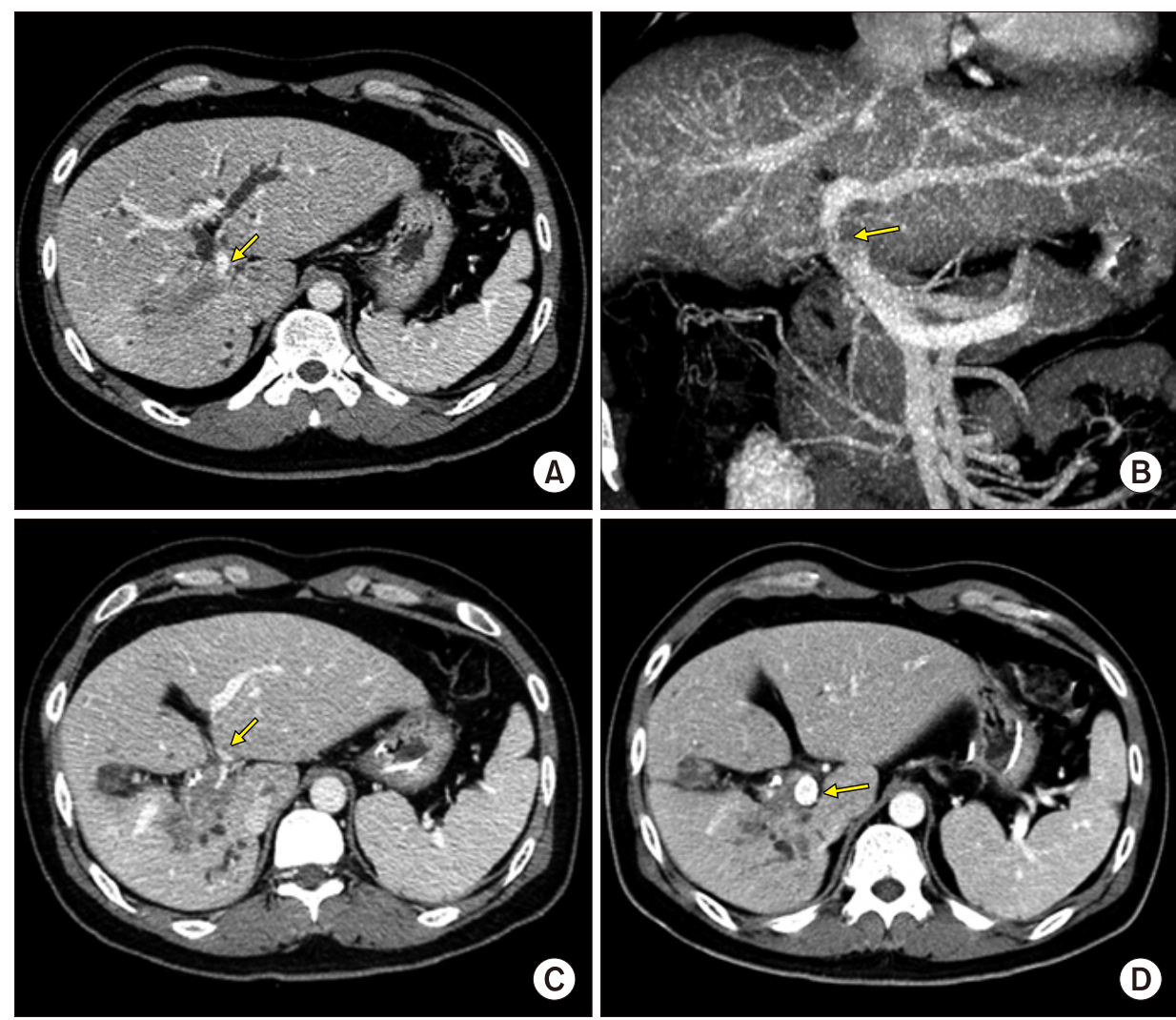

Fig. 1. Preoperative computed tomography findings. The portal vein was invaded by the tumor (A and B), progressively occluded (C), and expanded after stent insertion (D). Arrows indicate the sites of portal vein invasion.
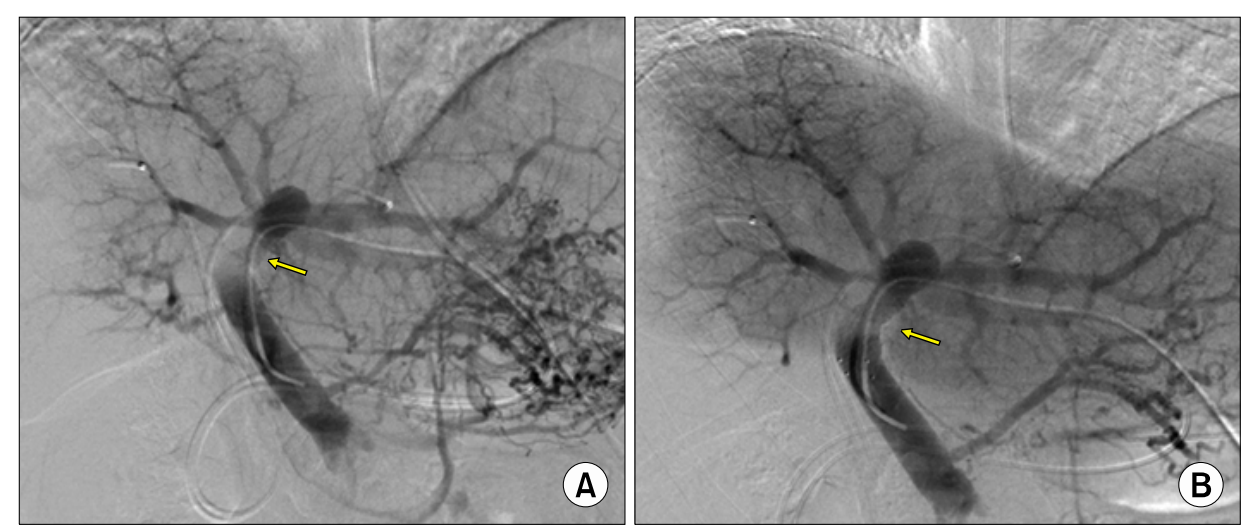

Fig. 2. Preoperative percutaneous portal vein stenting. The transverse portion of the left portal vein was stenotic (A) and expanded after insertion of a selfexpandable wall stent of $12 \mathrm{~mm} \times$ $6 \mathrm{~cm}$ in size (B). Arrows indicate the sites of portal vein invasion. 

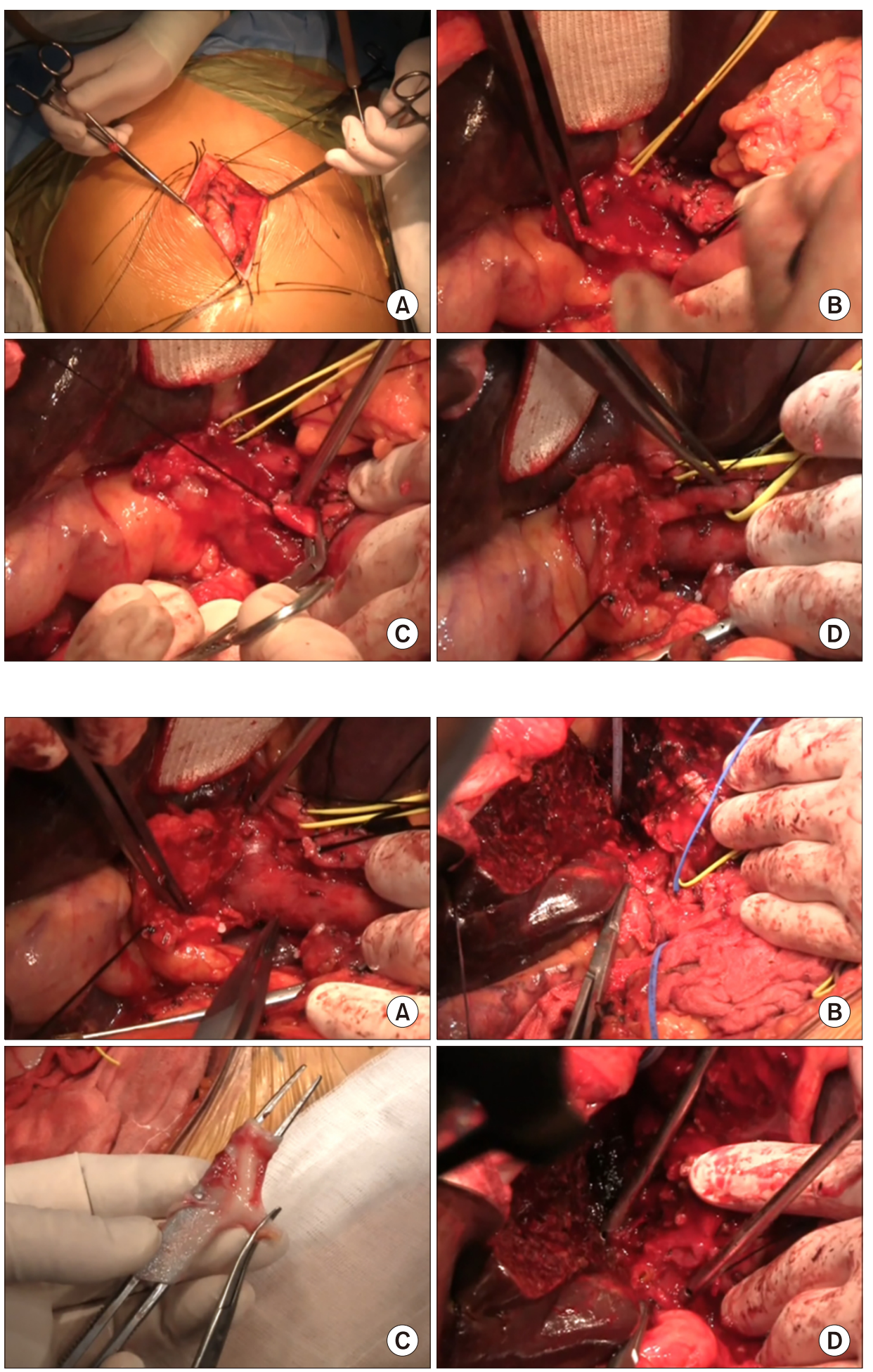

Fig. 3. Intraoperative photographs. (A) Abdominal exploration through mini-laparotomy. (B-D) dissection of the hepatoduodenal ligament. main PV was invaded by the tumor. After that, the left PV was dissected to assess whether to obtain tumor-free left hepatic duct resection margins and to determine whether the left PV stump was suitable for vascular anastomosis (Fig. 4A, B). After these assessment procedures, we finally decided to perform right trisectionectomy with en bloc PV resection and vein homograft interposition. A segment of cold-stored iliac vein homograft was prepared (Fig. 4C).

The liver parenchyma was transected along the falciform ligament and then the left hepatic duct was cut. Two bile duct openings from the segment II and III were ex- 
posed prepared (Fig. 4D). Intraoperative frozen-section biopsy revealed the left hepatic duct stumps were tumorfree. After meticulous dissection of the left PV branch close to the umbilical portion, the transverse portion of the left PV was transected (Fig. 5A). Since the stent was inserted deep into the umbilical portion of the left PV, a $1.5 \mathrm{~cm}$ long portion of the stent remained at the remnant left PV stump. The fragmented wire network of the disrupted wall stent was pulled out under loose clamping of the left PV stump by a vascular clamp (Fig. 5B). A $4 \mathrm{~cm}$-long iliac vein homograft was interposed with end-to-end anastomosis using 6-0 Prolene (Fig. 5C, D). After that, hepatic transection continued toward the dorsal part of the liver and the left caudate lobe was completely removed. After the right trisection liver specimen was delivered outside, extensive lymphadenectomy was performed from the posterior side of the pancreas to the celiac axis.

At this time, the main PV was abnormally distended with loss of blood flow on palpation. Intraoperative Doppler ultrasonography showed sluggish PV flow, implicating intrahepatic PV thrombus formation probably due to intimal damage induced by forceful extraction of the wall stent (Fig. 6A). After distal clamping of the interposed vein homograft (Fig. 6B), a Fogarty catheter was inserted through a small incision at the homograft (Fig. 6C) and a few small pieces of PV thrombus were removed (Fig. 6D). The surgical procedures were ended with Roux-Y hepaticojejunostomy for two left hepatic duct openings. Concern about further PV thrombus formation, a new wall stent was inserted intraoperatively through the inferior mesenteric vein to expand the homograft conduit (Fig. 7). The detailed surgical procedures were presented with a Supplementary video clip of 5 minutes.

The pathology report presented that the tumor was a $5.2 \mathrm{~cm}$-sized well-differentiated adenocarcinoma, thus being diagnosed as intrahepatic cholangiocarcinoma of periductal infiltrating type (Fig. 8). The tumor was extended to the periductal soft tissue and hepatic parenchyma. The main PV was involved with tumor. Lymphovascular invasion and perivascular invasion were present. There was metastasis in one of 3 regional lymph nodes. The left hepatic duct margins were tumor-free.

The patient recovered uneventfully and was discharged on the eleventh postoperative day. The patient underwent adjuvant chemotherapy because of the advanced tumor with extensive lymph node metastasis.

The PV stent was patent during the first year after surgery (Fig. 9). During postoperative follow-up, the interposed PV segment with a wall stent was gradually occluded with development of portal collaterals despite ad-
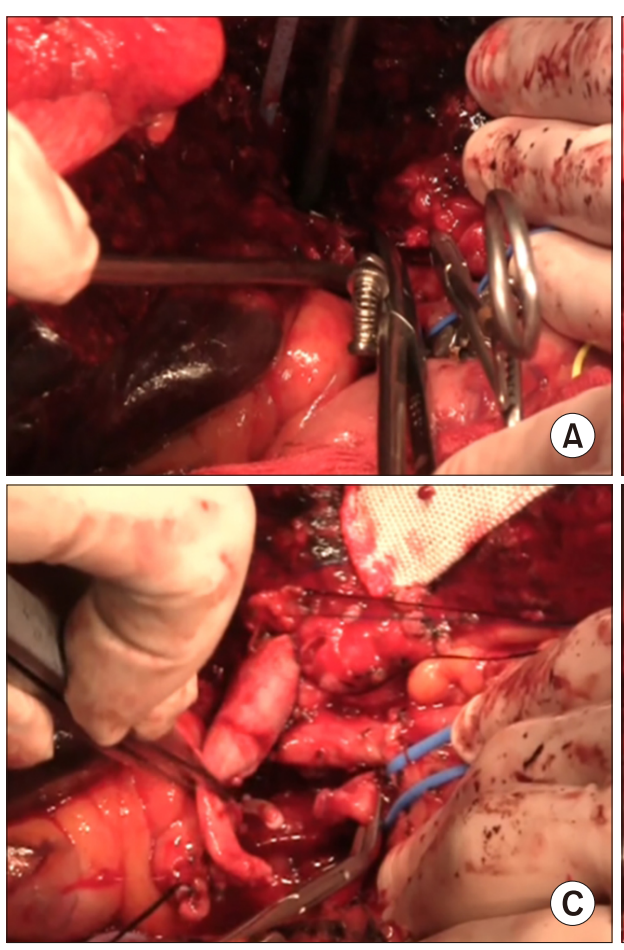

Fig. 5. Intraoperative photographs. (A) The transverse portion of the left portal vein was transected. (B) The fragmented wire network of the disrupted wall stent was removed from the left portal vein stump. (C and D) A $4 \mathrm{~cm}$-long iliac vein homograft was interposed with end-to-end anastomosis. 

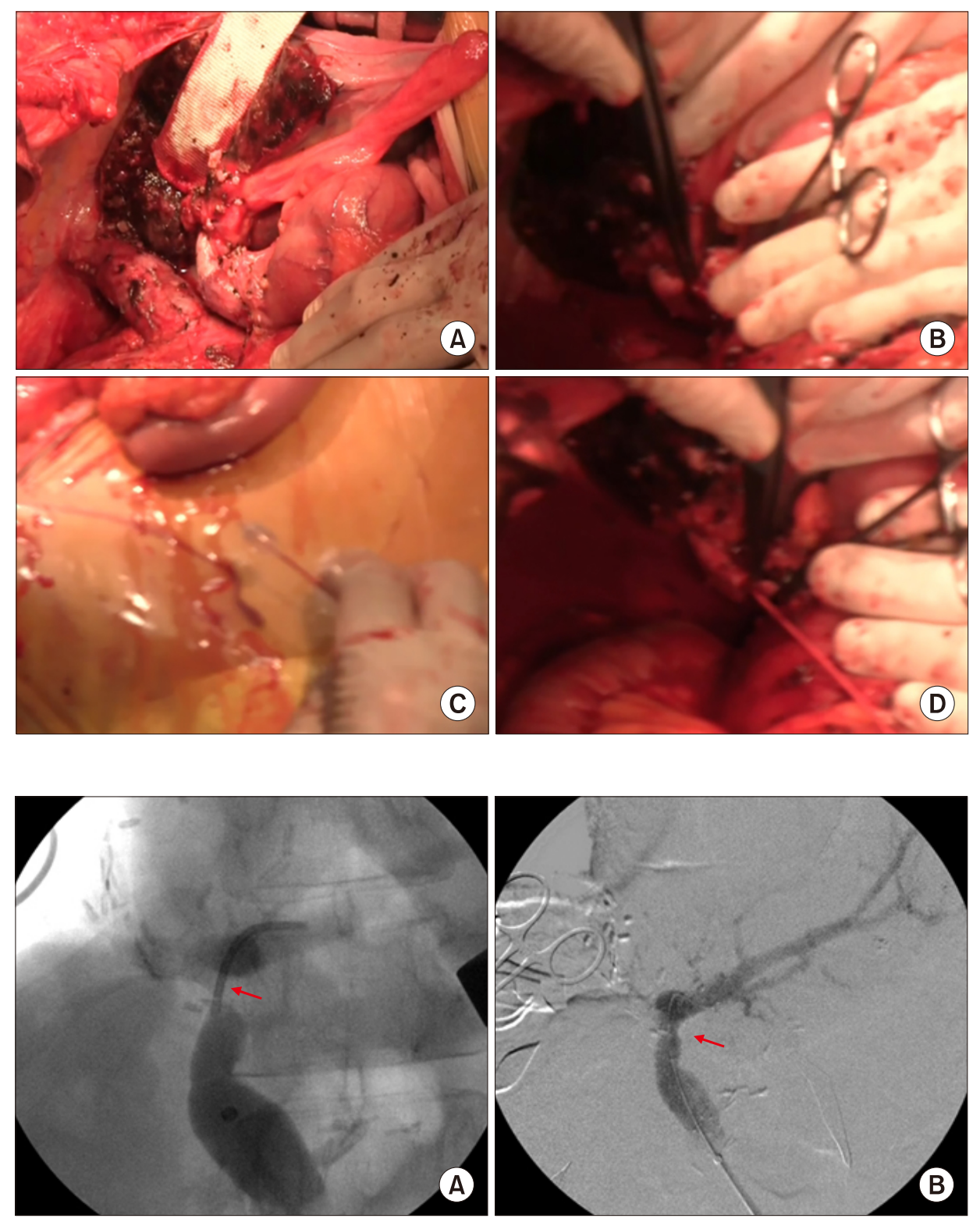

Fig. 7. Intraoperative portal vein stenting. The transverse and umbilical portion of the left portal vein was occluded (A) and expanded after insertion of a self-expandable wall stent of 12 $\mathrm{mm} \times 6 \mathrm{~cm}$ in size (B). Arrows indicate the sites of portal vein thrombosis.

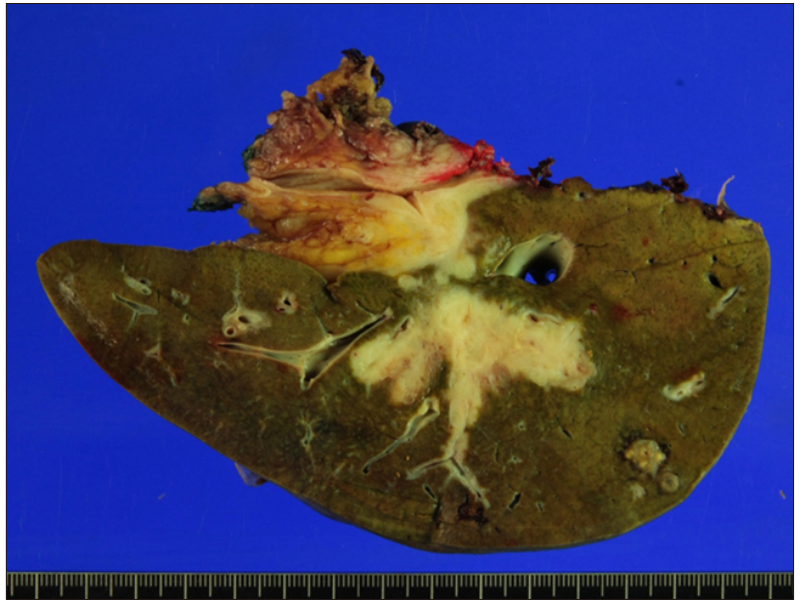

Fig. 8. Gross photograph of the resected specimen. ministration of low-dose aspirin. At 2 years after surgery, thrombus was formed within the PV stent, which induced PV stenosis. At 4 years after surgery, the PV stent was further occluded with thrombus, but intrahepatic PV flow was reconstituted through PV collateral formation (Fig. 10A). The intrahepatic ducts at the hepaticojejunostomy were stenotic with mild ductal dilatation, indicating benign stricture (Fig. 10B). At 5 years after surgery, the PV stent was completely occluded and cavernous transformation developed around the hepaticojejunostomy site (Fig. 10C, D).

The patient experienced repetition of febrile episodes of unknown causes. Ascending cholangitis was suspected for 

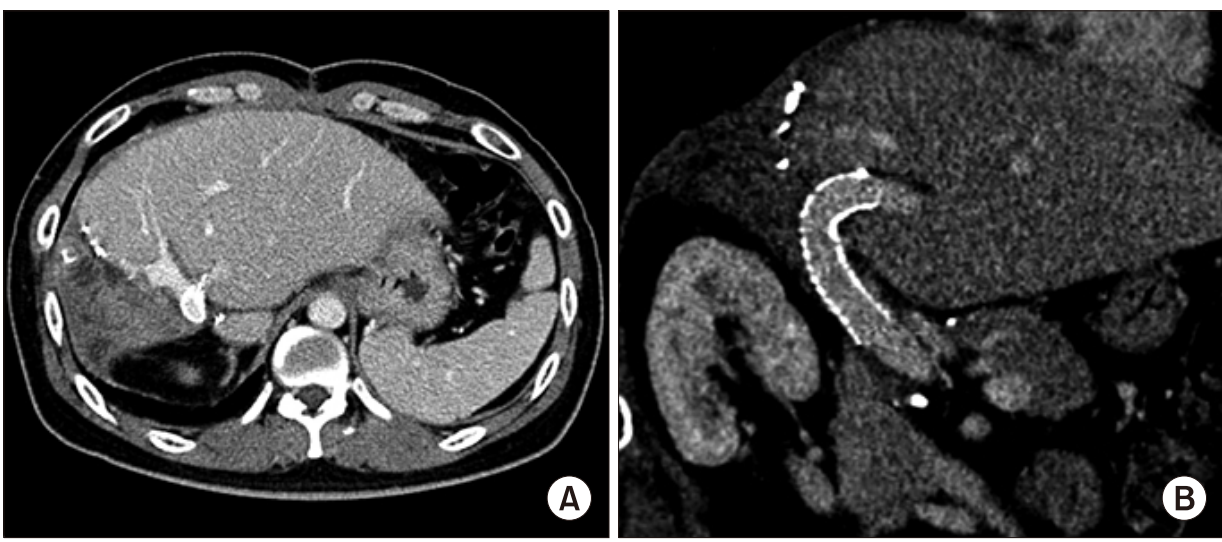

Fig. 9. Postoperative computed tomography findings taken 1 month after surgery. The portal vein conduit was patent on the axial image (A) and reconstructed image (B).
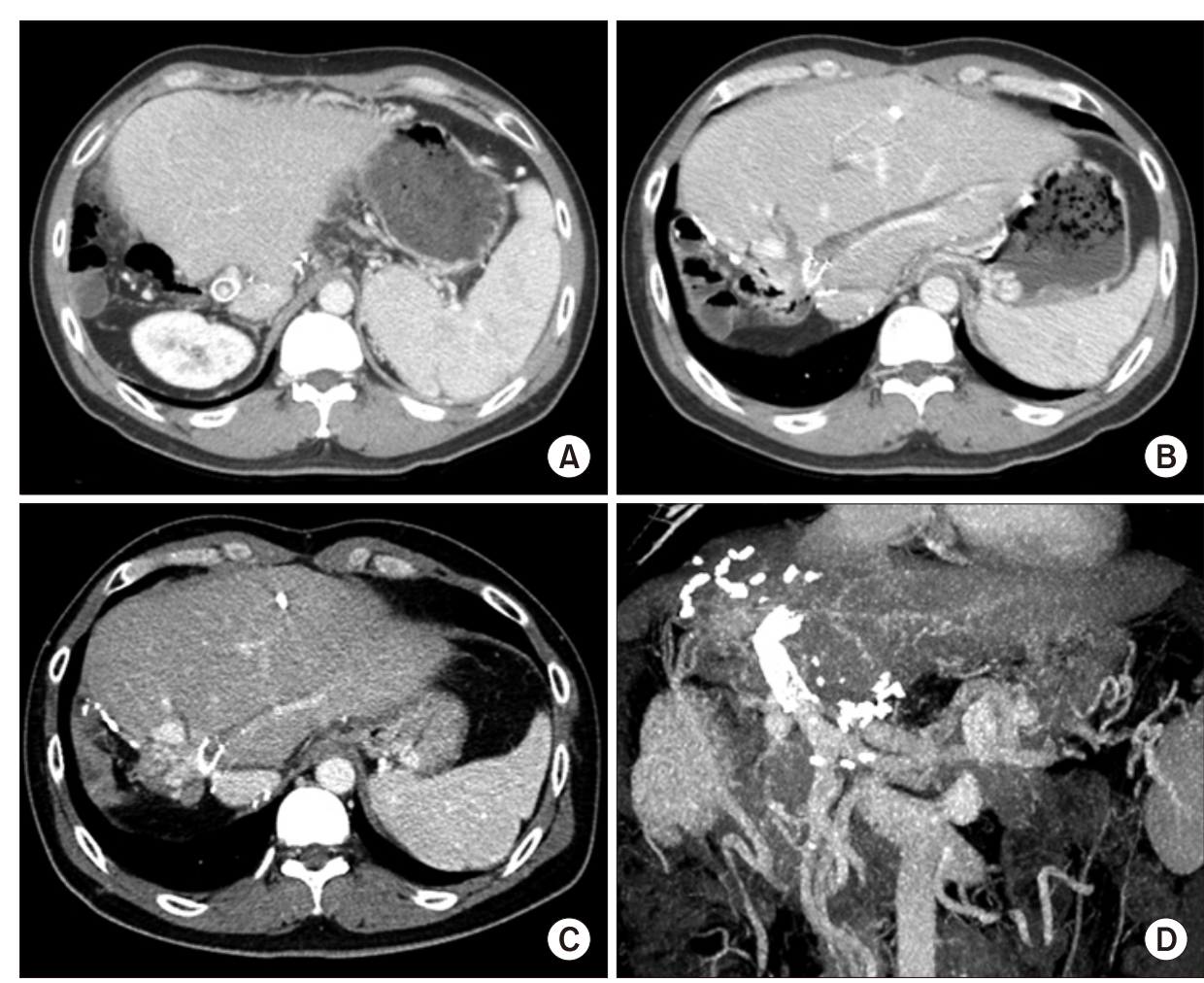

Fig. 10. Postoperative computed tomography follow-up. (A and B) At 4 years after surgery, the portal vein stent was partially occluded with thrombus and the intrahepatic ducts at the hepaticojejunostomy were stenotic with mild ductal dilatation. ( $\mathrm{C}$ and D) At 7 years, the portal vein stent was completely occluded with cavernous transformation and collaterals were developed.

the underlying cause of fever, but biliary excretion rates at 90 minutes remained at approximately $90 \%$ on hepatobiliary scintigraphy and there was no abnormality in the liver function test. He is currently alive for 8 years with no evidence of tumor recurrence.

\section{DISCUSSION}

Advanced perihilar cholangiocarcinoma and central-type intrahepatic cholangiocarcinoma can invades the PV, resulting in PV stenosis and a decrease in hepatopetal portal flow. Such PV invasion is usually accompanied by obstructive jaundice from hilar bile duct obstruction. Deprivation of PV blood flow hinders functional recovery of the liver function from obstructive jaundice. As shown in this case, resolution of obstructive jaundice was unusually slow despite external biliary drainage under PV occlusion. If obstructive jaundice persists, the patient is not indicated for major hepatectomy because of high risk of post-hepatectomy liver failure. In contrast, after restoration of PV flow through PV stenting, obstructive jaundice was resolved rapidly. These findings indicate the importance of adequate PV blood flow during recovery of liver function and resolution of obstructive jaundice. 
To our knowledge, there were only two reports on preoperative PV embolization after preoperative PV stenting to cope with severe PV tumor invasion and stenosis, which were one case of gallbladder cancer ${ }^{1}$ and 4 cases of perihilar cholangiocarcinoma. ${ }^{2}$ In this case, PV embolization was unnecessary because the right PV was already occluded by tumor invasion and compensatory hypertrophy was already developed at the future remnant left liver.

The indications for percutaneous PV stenting include the treatment of prehepatic portal hypertension associated with PV stenosis, such as varices of portal collateral vessels, refractory ascites, pancytopenia and liver dysfunction due to advanced or recurrent pancreaticobiliary tumor., ${ }^{3,4}$ Yamakado et al. ${ }^{5}$ reported that 40 patients, including 23 cases of hepatocellular carcinoma, 9 cases of pancreatic cancer, and 8 cases of bile duct cancer, underwent percutaneous PV stenting. They concluded that splanchnic vein involvement, severe hepatic dysfunction, and obstruction of the portal venous system are the most crucial factors affecting stent occlusion in patients with malignant PV invasion.

Intraoperative and postoperative PV stenting can be performed in patients undergoing PV reconstruction during liver transplantation and hepatobiliary surgery if anastomotic PV stenosis occurs. ${ }^{6-10}$ Zhou et al. ${ }^{6}$ reported that the reasons for PV stent placement in 59 patients with hepatobiliary and pancreatic cancers in our institution were tumor recurrence $(n=30), P V$ resection and anastomosis $(n=18)$ and postoperative inflammatory changes $(n=11)$. We previously reported a case of PV stenting to treat gastrointestinal bleeding secondary to PV stenosis caused by the local recurrence of the distal bile duct cancer after pancreatoduodenectomy. ${ }^{10}$

Unlike all cases that have undergone PV stenting in the literature, the unique feature of our case is that the first PV stent was removed completely because the PV stent was encased by the tumor. In the literature, there was no report on intraoperative en bloc removal of the PV stent, thus our study would be the first case report. Because of this reason, we presented a supplementary video clip to present the detailed surgical procedures. The 3-dimensional structure of a self-expandable wall stent is disrupted when it is transected midway, by which the stent wires can be extracted under loose clamping. We experienced similar extraction of stent wires during liver trans- plantation in patients who had undergone transjugular intrahepatic portosystemic shunt. Since the wall stent wires were located deeply at the umbilical portion of the left $\mathrm{PV}$ in this case, its removal induced intimal damage of the remnant left PV. Such intimal injury was thrombogenic, thus unexplainable early PV thrombus developed during operation. We were well experienced with vascular reconstruction because of hundreds cases of liver transplantation per year, thus technical fault was not taken into account. The intrahepatic PV thrombus was extracted and removed with a Fogarty catheter. To secure PV flow, a new wall stent was inserted to expand the homograft conduit during the operation.

The patient is currently alive for 8 years without tumor recurrence after aggressive surgery although the tumor was far advanced and lymph node metastasis was present. We believe that the patient definitely benefited from preoperative PV stenting because it enabled him to undergo major hepatectomy. Our experience will be helpful to decide surgical planning for hepatobiliary malignancy patients with PV invasion.

\section{SUPPLEMENTARY DATA}

Supplementary data related to this article can be found at https://doi.org/10.14701/ahbps.2020.24.2.174.

\section{CONFLICT OF INTEREST}

None of the authors has any conflict of interest.

\section{ORCID}

Shin Hwang: https://orcid.org/0000-0002-9045-2531

Gi-Young Ko: https://orcid.org/0000-0003-4617-1799

\section{AUTHOR CONTRIBUTIONS}

Conceptualization: SH, GYK. Data curation: SH, GYK. Visualization: SH. Writing - original draft: SH. Writing review \& editing: $\mathrm{SH}$.

\section{REFERENCES}

1. Kaneoka Y, Yamaguchi A, Isogai M, Hori A. Intraportal stent 
placement combined with right portal vein embolization against advanced gallbladder carcinoma. Surg Today 1998;28:862-865.

2. Hyodo R, Suzuki K, Ebata T, Komada T, Mori Y, Yokoyama $\mathrm{Y}$, et al. Assessment of percutaneous transhepatic portal vein embolization with portal vein stenting for perihilar cholangiocarcinoma with severe portal vein stenosis. J Hepatobiliary Pancreat Sci 2015;22:310-315.

3. Novellas S, Denys A, Bize P, Brunner P, Motamedi JP, Gugenheim J, et al. Palliative portal vein stent placement in malignant and symptomatic extrinsic portal vein stenosis or occlusion. Cardiovasc Intervent Radiol 2009;32:462-470.

4. Woodrum DA, Bjarnason H, Andrews JC. Portal vein venoplasty and stent placement in the nontransplant population. J Vasc Interv Radiol 2009;20:593-599.

5. Yamakado K, Nakatsuka A, Tanaka N, Fujii A, Terada N, Takeda K. Malignant portal venous obstructions treated by stent placement: significant factors affecting patency. J Vasc Interv Radiol 2001;12:1407-1415.
6. Zhou ZQ, Lee JH, Song KB, Hwang JW, Kim SC, Lee YJ, et al. Clinical usefulness of portal venous stent in hepatobiliary pancreatic cancers. ANZ J Surg 2014;84:346-352.

7. Ko GY, Sung KB, Yoon HK, Lee S. Early posttransplantation portal vein stenosis following living donor liver transplantation: percutaneous transhepatic primary stent placement. Liver Transpl 2007;13:530-536.

8. Kim YJ, Ko GY, Yoon HK, Shin JH, Ko HK, Sung KB. Intraoperative stent placement in the portal vein during or after liver transplantation. Liver Transpl 2007;13:1145-1152.

9. Ohm JY, Ko GY, Sung KB, Gwon DI, Ko HK. Safety and efficacy of transhepatic and transsplenic access for endovascular management of portal vein complications after liver transplantation. Liver Transpl 2017;23:1133-1142.

10. Hwang S, Sung KB, Park YH, Jung DH, Lee SG. Portal vein stenting for portal hypertension caused by local recurrence after pancreatoduodenectomy for periampullary cancer. J Gastrointest Surg 2007;11:333-337. 